Supporting Information for

\title{
From mechanism to device in polymer-assisted rubrene crystallization
}

Ilya Bronshtein, Haim Weissman, Idan Baran, and Boris Rybtchinski*

Department of Molecular Chemistry and Materials Science, Weizmann Institute of Science, Rehovot, Israel.

* boris.rybtchinski@weizmann.ac.il 


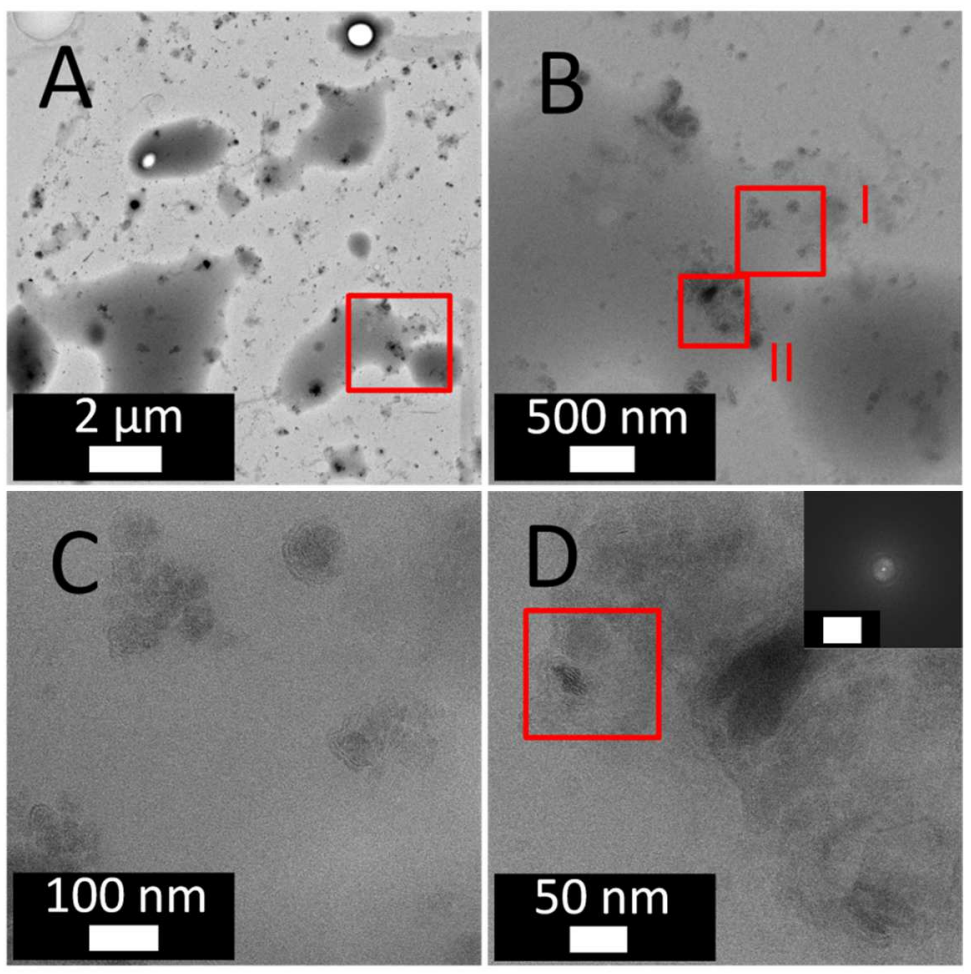

Figure S1. (A) TEM image of rubrene films after $20 \mathrm{~s}$ heat-plate treatment at $250{ }^{\circ} \mathrm{C}$ in $\mathrm{Ar}$ environment. (B) Magnified view of the marked area in the main images. (C) Magnified view of the marked area I in A. (D) Magnified view of the marked area II in B. Inset scale bars are $2 \mathrm{~nm}^{-1}$.
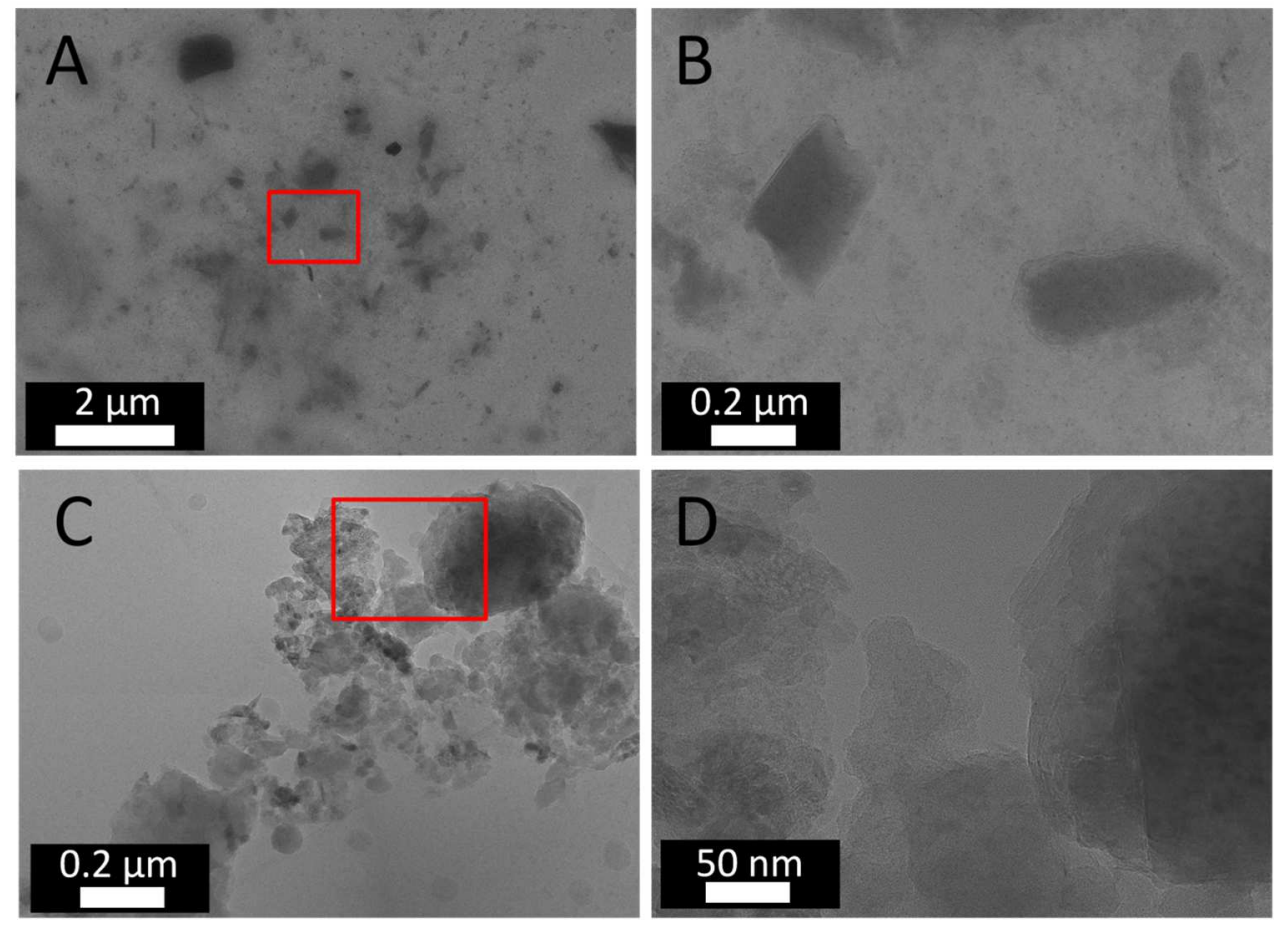

Figure S2. (A,C) TEM images of rubrene films after $300 \mathrm{~s}$ heat-plate treatment at $250{ }^{\circ} \mathrm{C}$ in Ar environment. (B,D) Magnified views of the marked area in the main images. 

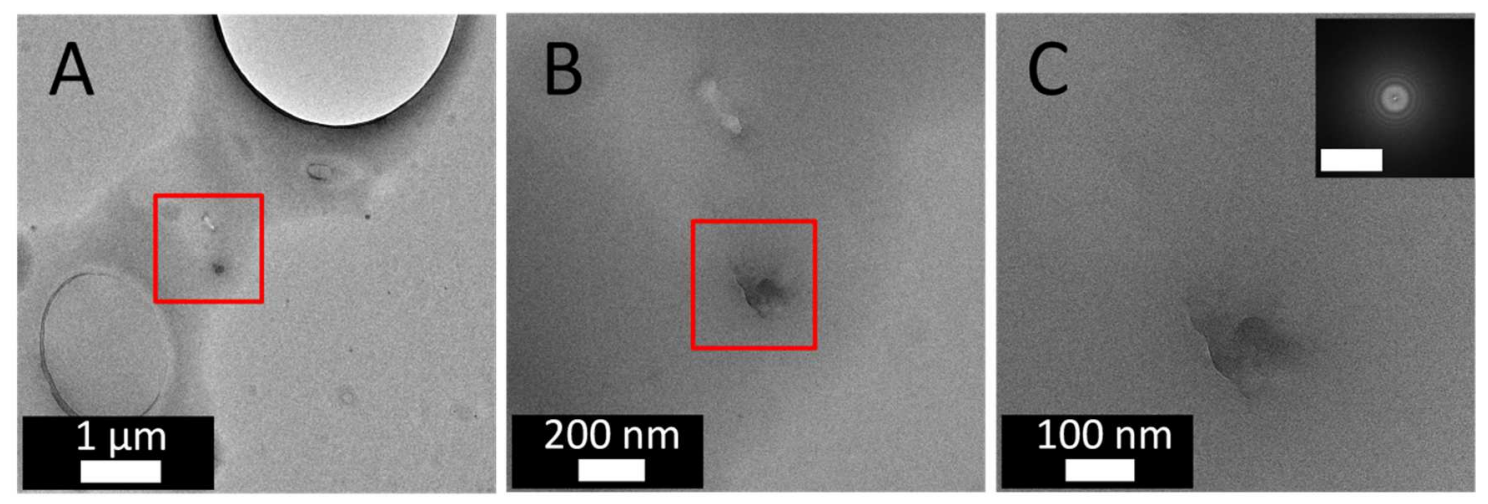

Figure S3. TEM images obtained immediately after Rub/PS film deposition (no heating). (A) Low magnification TEM images. (B) Magnified view of the marked area in A. (C) Magnified view of the marked area in $\mathrm{B}$. Inset scale bars are $2 \mathrm{~nm}^{-1}$

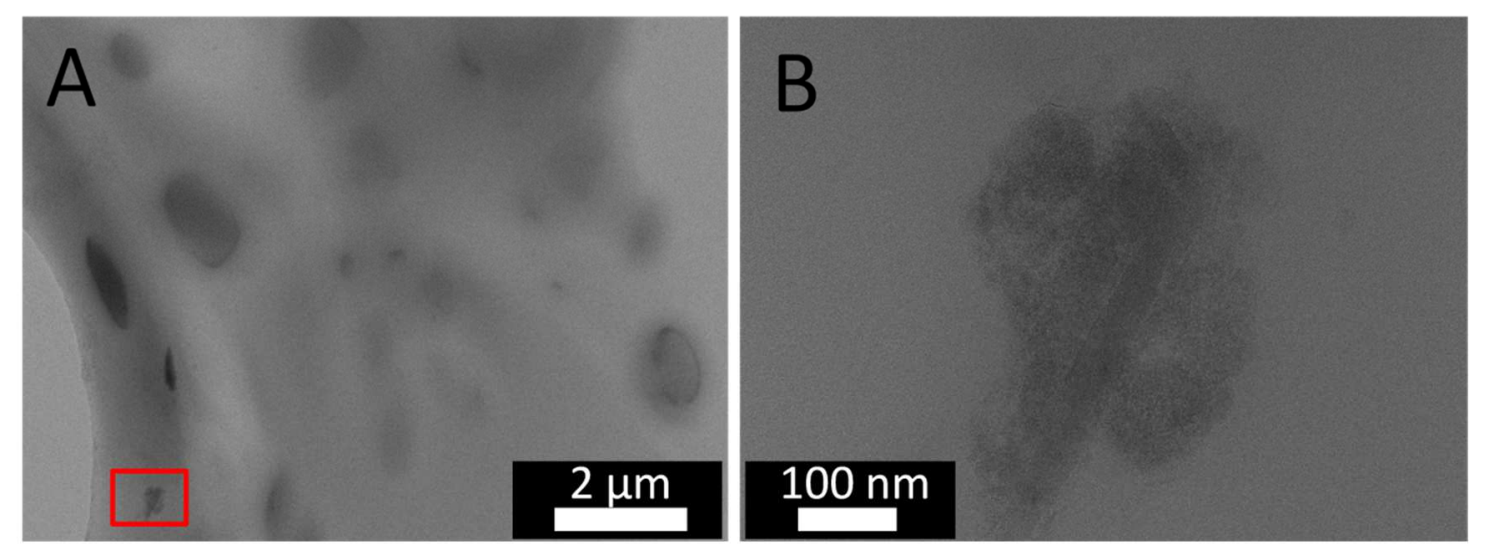

Figure S4. TEM images of rubrene films after $1 \mathrm{~s}$ of RTP treatment at $250{ }^{\circ} \mathrm{C}$. (A, C) Low magnification TEM images. (B) Magnified view of the marked area in A.
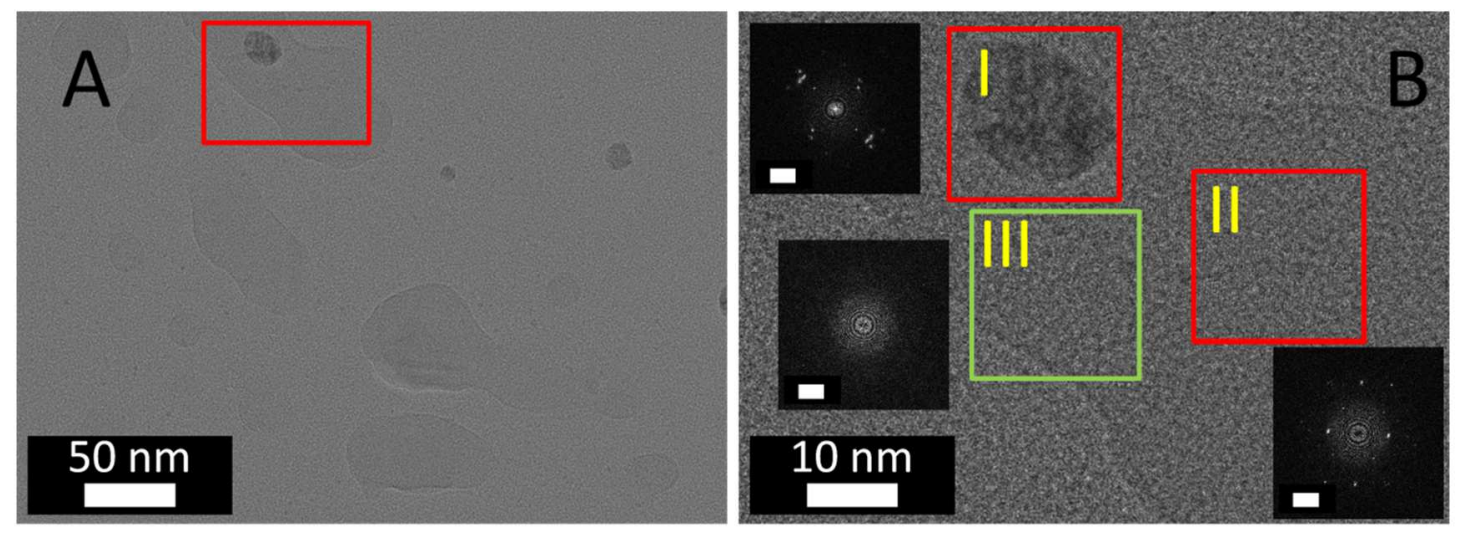

Figure S5. (A) TEM images of rubrene films after $5 \mathrm{~s}$ of RTP treatment at $250{ }^{\circ} \mathrm{C}$. B) Magnified view of the marked area in A. Insets: FFTs. Inset scale bars are $2 \mathrm{~nm}^{-1}$. 

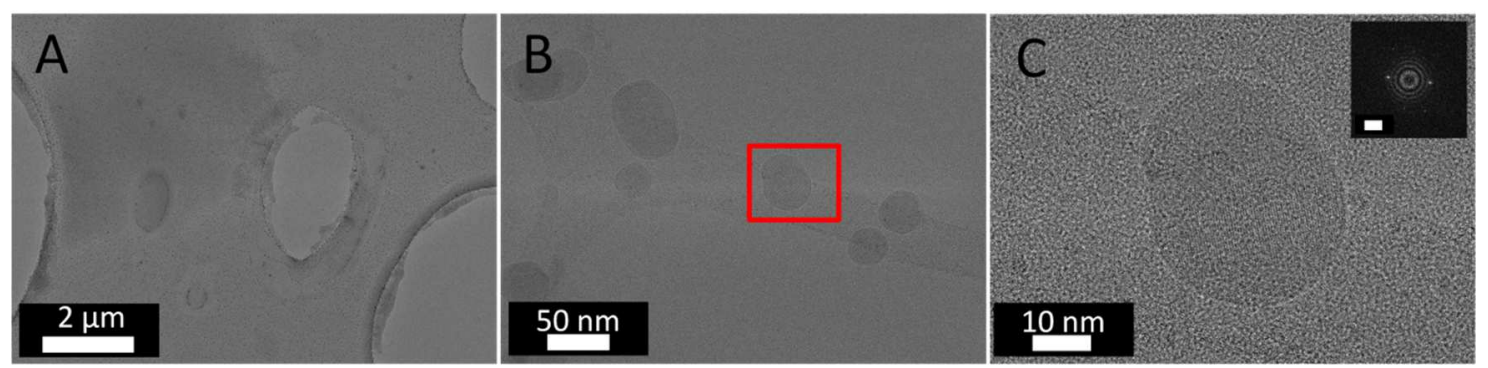

Figure S6. TEM images of rubrene films after $20 \mathrm{~s}$ of RTP treatment at $250{ }^{\circ} \mathrm{C}$. (A) Low magnification TEM image. (B) Magnified view. (C) Magnified view of the marked area in B. Inset: FFT of the image in $\mathrm{C}$.

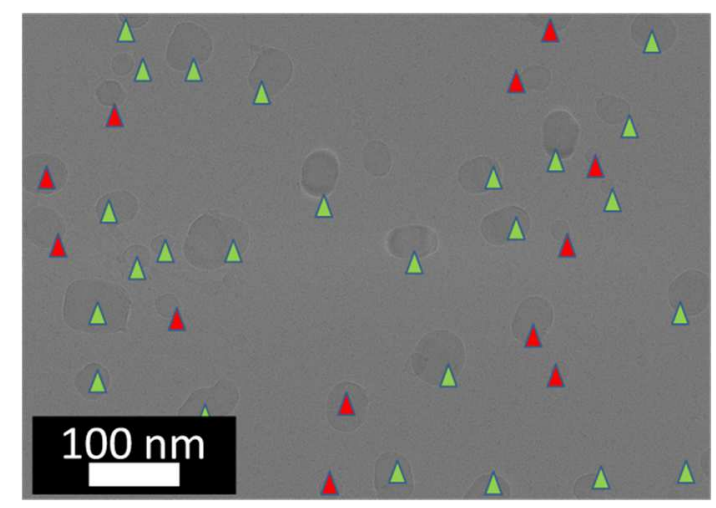

Figure S7. TEM image of rubrene films after $20 \mathrm{~s}$ of RTP treatment at $270^{\circ} \mathrm{C}$, crystalline ovals are marked by green triangles (FFT observed); the ovals where FFT was not observed are marked by red triangles. 


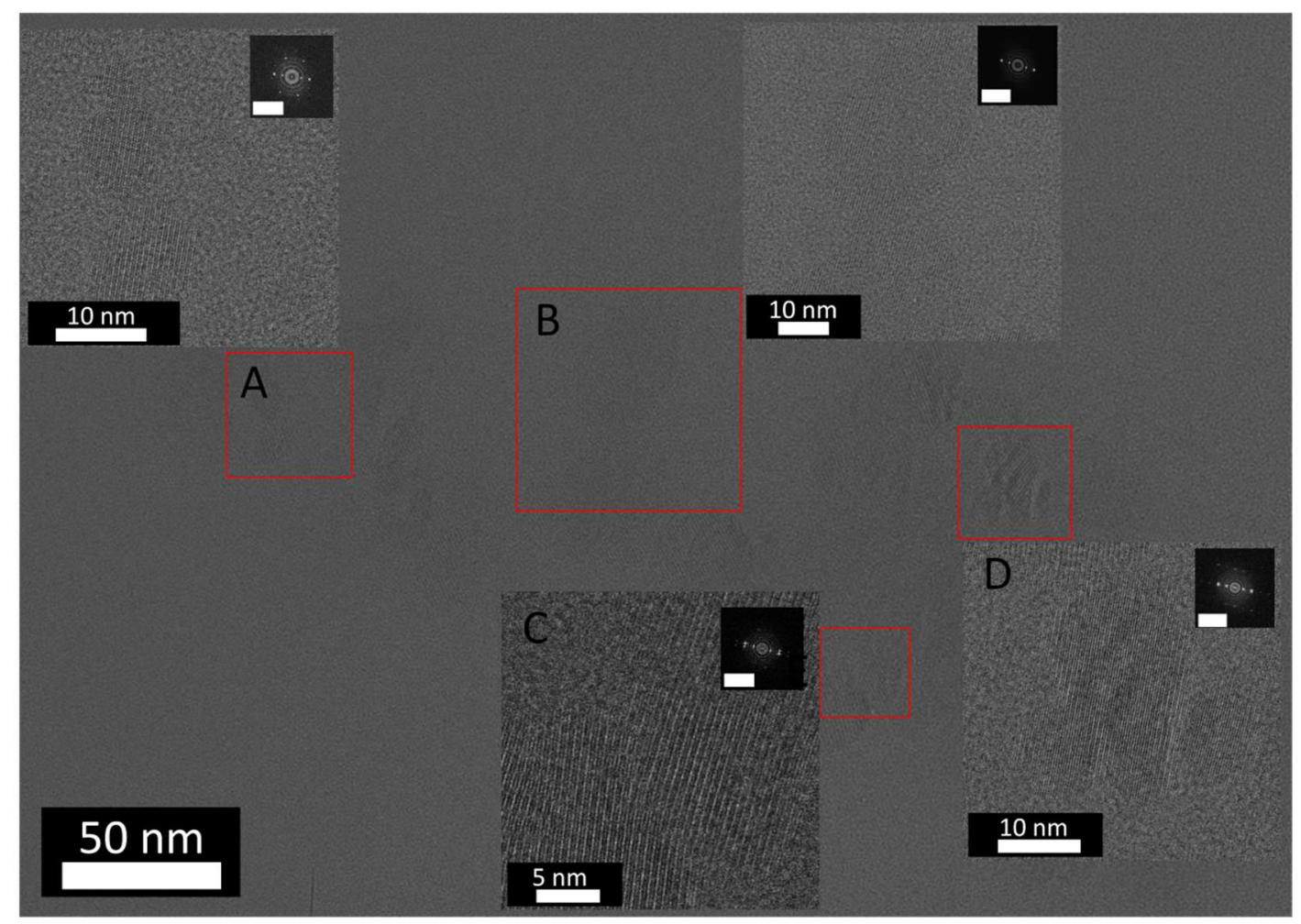

Figure S8. TEM images of rubrene films after $300 \mathrm{~s}$ of RTP treatment at $250{ }^{\circ} \mathrm{C}$. (A-D)

Magnified views of the marked area in the main image. Inset: FFTs indicating 2.09, 3.14, 6.28 $\AA$, and $2.89 \AA$ A spacings. Scale bars are $5 \mathrm{~nm}^{-1}$.
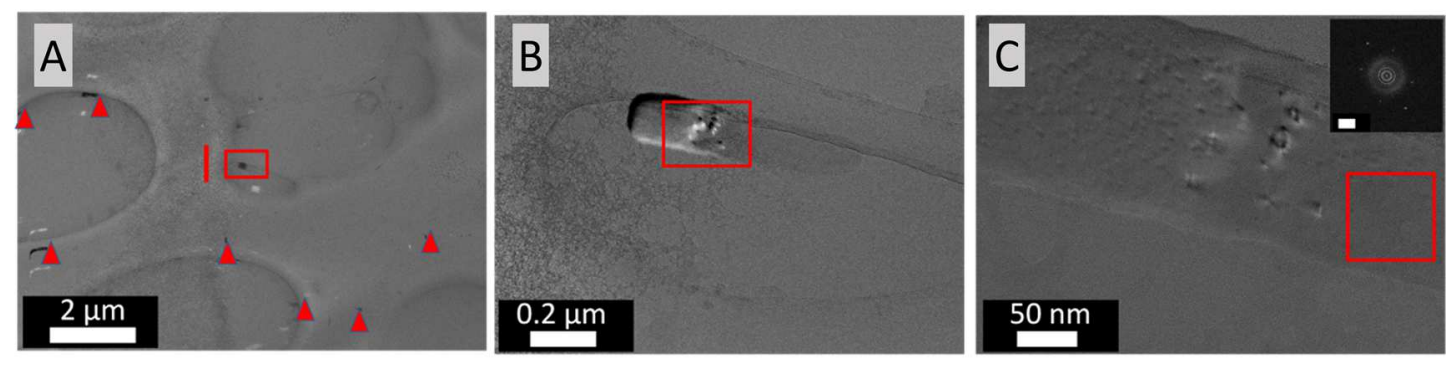

Figure S9. TEM images of rubrene films after $300 \mathrm{~s}$ of RTP treatment at $270{ }^{\circ} \mathrm{C}$. (A) Low magnification TEM image. (B) Magnified view of the marked area in A. (C) Magnified view of the marked area in B. FFTs indicating 3.05, 2.88 and $2.07 \AA$ spacings. Scale bars are 5 $\mathrm{nm}^{-1}$. 

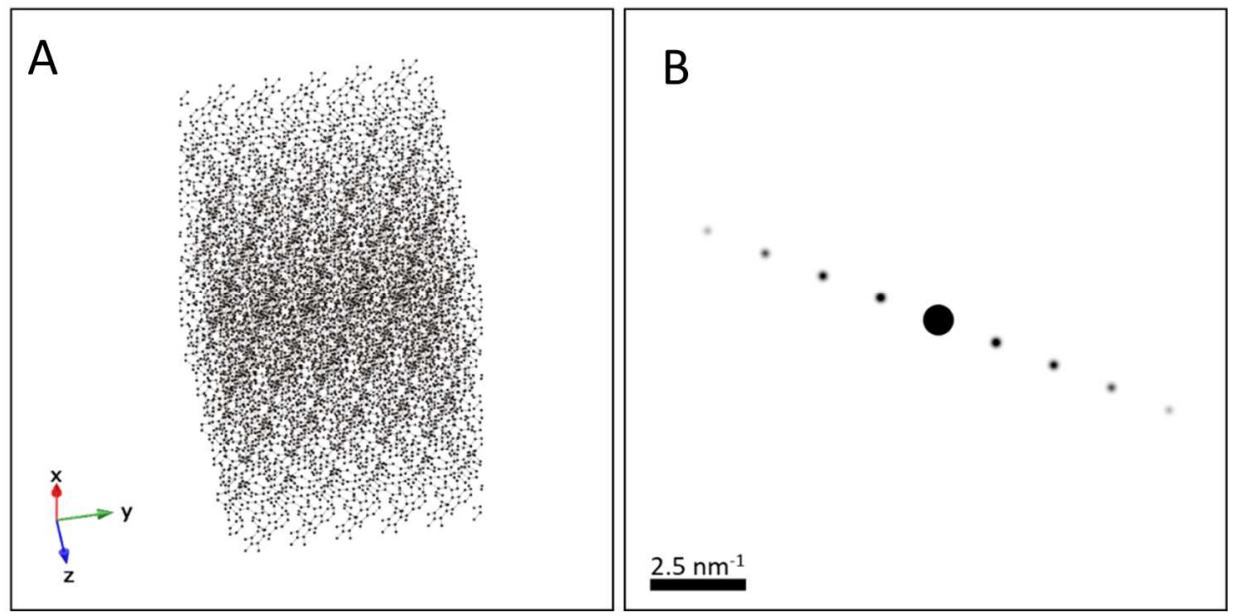

Figure S10. Rubrene crystal orientation ((111), based on structure CCDC 605647) that is the best match for the crystal orientation observed in Figure 7 (main text). (A) Structure in viewing direction of UVW vectors [0.089 -0.048 0.138], hydrogens are omitted for clarity. (B) simulated electron diffraction pattern (from panel A). Both the structure orientation and simulated diffraction fit the image and FFT observed in Figure 7.

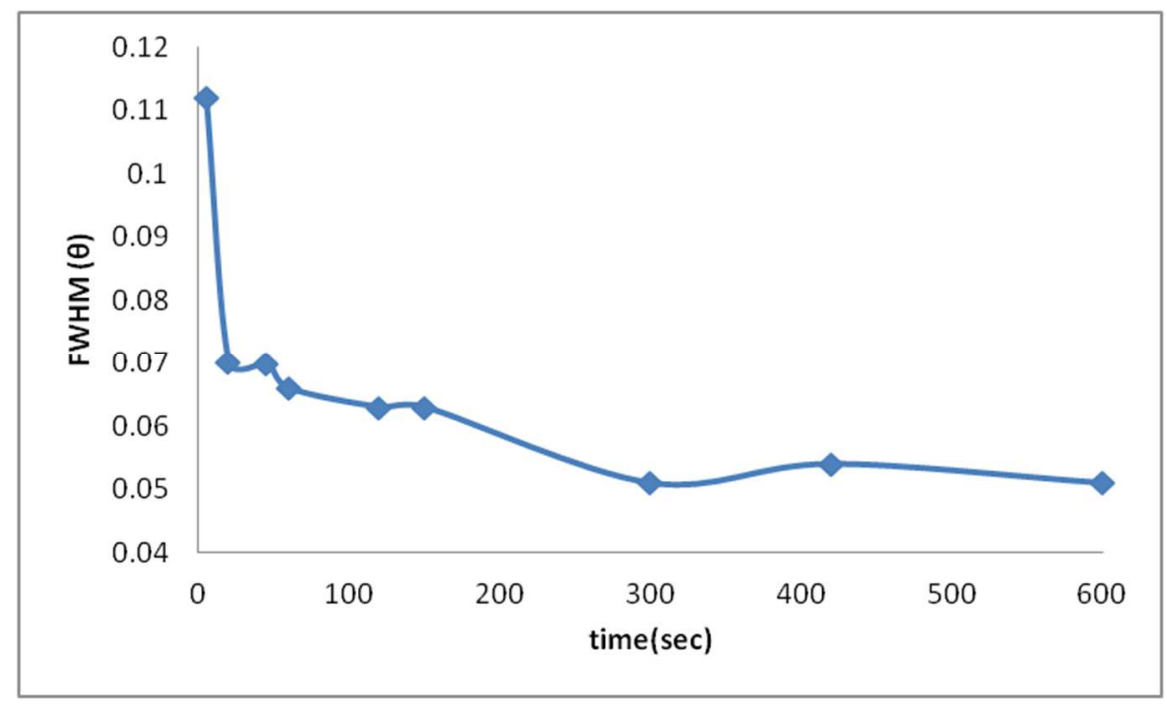

Figure S11. Time-dependent changes in peak broadening (Full Width at Half Maximum, FWHM) obtained after RTP treatment at $250^{\circ}$ of Rubrene/PS film deposited by spin-coating on untreated $\mathrm{Si} / \mathrm{SiO}_{2}$ surface. Changes in FWHM are concomitant with the evolution of average size of crystals, consistent with the TEM study. 